\title{
LA FORMACIÓN DEL FUTURO DOCENTE A TRAVÉS DE LA INTERDISCIPLINARIEDAD Y LA EDUCACIÓN INTEGRAL
}

\section{THE TRAINING OF THE TEACHING FUTURE THROUGH INTERDISCIPLINARITY AND INTEGRAL EDUCATION}

Fecha recepción: 1 de septiembre de 2019 / fecha aceptación: 1 de diciembre de 2020

\section{Luz Mㅁ Gilabert González y Mㅁ del Mar Bernabé Villodre ${ }^{2}$}

\section{Cómo citar este artículo:}

Gilabert, L. y Bernabé, M. (2020). Propuesta de intervención de trabajo social territorial con comunidades rurales de chile central en contexto de globalización. Revista Pensamiento y Acción Interdisciplinaria, 6(2), 104-120. http://doi.org/10.29035/pai.6.2.104

\section{Resumen}

La Expresión Plástica y la Expresión Musical tienen una especial relevancia para la formación integral del alumnado de Educación Infantil. La formación en o desde estas áreas de conocimiento debe continuarse en la siguiente etapa, Educación Primaria, mediante el estudio de la denominada Área de Educación Artística (comprende las materias de Plástica y Música). El futuro profesorado de Educación Infantil debe plantearse la formación en Educación Artística no sólo como un todo interrelacionado que garantice la formación integral del alumnado de dicha etapa educativa, sino como herramientas que favorecerán y potenciarán el desarrollo de todas las áreas fundamentales (cognitiva, motora, lingüística, etc.). Este artículo presenta los resultados de una propuesta interdisciplinar desarrollada en el tercer curso del Grado de Educación Infantil de una universidad española, entre cuatro asignaturas, a través de los lenguajes plástico, visual y musical. Las actividades propuestas combinaron lengua castellana, teatro, música, arte y TIC. Esta experiencia permitió demostrar la importancia del trabajo interdisciplinar durante la formación de los futuros maestros en Educación Infantil y las infinitas posibilidades que existen a la hora de plantear ejercicios que fomenten la educación integral y el trabajo colaborativo entre diferentes disciplinas.

Palabras clave: Creatividad, Educación Artística, Educación Infantil, Interdisciplinariedad, Música, Plástica.

\footnotetext{
1 Española. Funcionaria de Carrera del Cuerpo de Profesores de Educación Secundaria. Doctora con mención europea en Historia del Arte por la Universidad de Murcia. Instituto de Educación Secundaria Los Montesinos-Remedios Muñoz (Alicante). Comunidad Valenciana, España. Correo electrónico: luz.gilabert@gmail.com

2 Española. Profesora universitaria. Doctora en Teoría e Historia de la Educación por la Universidad de Murcia. Universidad de Valencia. Comunidad Valenciana, España. Correo electrónico: maria.mar.bernabe@uv.es
} 


\begin{abstract}
The Plastic Expression and the Musical Expression have a special relevance for the integral formation of the students of Early Childhood Education. Training in or from these areas of knowledge should be continued in the next stage, Primary Education, through the study of the so-called Area of Artistic Education (includes the subjects of Plastic and Music). The future teachers of Early Childhood Education should consider training in artistic education not only as an interrelated whole that guarantees the integral formation of the students of this educational stage, but as tools that favor and enhance the development of all fundamental areas (cognitive, motor, linguistics, etc.). This article presents the results of an interdisciplinary proposal developed in the third year of the Degree of Infantile Education of a Spanish university, between four subjects, through the plastic, visual and musical languages. The proposed activities combined Spanish language, theater, music, art and ICT. This experience allowed us to demonstrate the importance of interdisciplinary work during the training of future teachers in Early Childhood Education and the infinite possibilities that exist when proposing exercises that promote integral education and collaborative work between different disciplines.
\end{abstract}

Keywords: Arts Education, Creativity, Early Childhood Education, Interdisciplinarity, Music, Plastic Arts.

\title{
Introducción
}

De acuerdo con el Real Decreto 1630/2006, de 29 de diciembre, por el que se establecen las enseñanzas mínimas del segundo ciclo de Educación Infantil, el docente de Educación Infantil debe desarrollar las siguientes áreas de desarrollo: área de conocimiento de sí mismo y autonomía personal, área de conocimiento del entorno, y área de comunicación y representación. Será en esta última donde se encuentren situadas las materias de Plástica y de Música como lenguajes expresivos de comunicación emocional, con mucho más que aportar si se incorporan de forma transdisciplinar a los distintos momentos de trabajo en el aula de Educación Infantil. Su visión como herramientas de mejora ya ha sido comentada por distintos especialistas, a través de experiencias interdisciplinares en distintas etapas educativas: por ejemplo, Pérez y Leganés (2012), Chao, Mato y López (2015), Gilabert y Bernabé (2016), entre otros.

A estas áreas incluidas en la legislación española para Educación Infantil, vendrían a sumarse las tradicionales áreas de desarrollo del niño de 0 a 6 años, ampliamente comentadas por autores como Piaget (1990), y que podemos concretar en: área física, área de lenguaje, área cognoscitiva y área socioafectiva. La consecución de todas ellas lleva al educando a conseguir la ansiada educación integral; de manera que, el futuro docente necesita ser formado con esa visión globalizada de las aportaciones y conjunciones de cada materia en la siguiente. Para esto, es necesario proponer al docente en formación un trabajo interdisciplinar, que le permita comprender la necesidad de interrelacionar cada uno de los campos de conocimiento estudiados; pero, esto sólo será posible (y factible) desde la unión de varias materias y especialistas a lo largo del mismo curso académico como serían la Música, la Lengua Castellana y la Psicología del Desarrollo. 
La formación del futuro docente a través de la interdisciplinariedad y la educación integral por Luz Ma Gilabert González y Ma del Mar Bernabé Villodre

\section{Marco referencial}

\section{Educación integral: breve aproximación al concepto desde las artes}

Si se parte de las concepciones de la Antigüedad Clásica, una formación integral venía a representar lo contrario a una formación filosófica, por ejemplo. Educación integral, entonces, haría referencia a una educación que supusiera la formación total del individuo; aunque, como individuo que debe educarse, nunca como comunidad que debe educarse (Rodríguez, 2012), pero no entraremos en ello porque supondría una discusión diferente. Lo que sí debemos tener en cuenta es que, más allá de las diferentes interpretaciones del concepto, la educación integral incluye el concepto de totalidad (Gevilla, 2000), esto es, la educación individualizada del ser humano en cada una de sus dimensiones.

Barrientos (2013) considera que la visión integral de la educación comprende una intencionalidad permanente, extendida a lo largo de la vida. Aquí, podemos comprobar muchas similitudes con el proceso educativo artístico (bien plástico y/o musical), que parte de la acumulación de conceptos-procedimientos en distintos estadios que se van sumando y son imprescindibles para el paso al siguiente estadio artístico.

Lo que debemos tener en cuenta es que cuando se educa integralmente, se parte del trabajo de conocimientos y de habilidades que puedan estar (o no) inherentes en el alumnado. Así, de acuerdo con López-Peláez (2010), conocimiento y habilidades podrían considerarse sinérgicos, siendo esta interpretación la que posibilita que los beneficios que se obtienen al interrelacionarse distintas materias, sea mucho mayor que los aprendizajes de materias separadamente. Estas sinergias de contenidos-habilidades son mucho más habituales entre materias de la familia de las Artes, como son la Música y la Plástica, por ejemplo; pero, no por ello, deberían considerarse totalmente alejadas del resto de manifestaciones intelectuales del ser humano. Es decir, que un proceso de enseñanza/aprendizaje en Educación Infantil será más completo cuanto más interdisciplinarmente se trabaje: sólo la concepción integral de la educación posibilitará esto.

\section{La importancia de la interdisciplinariedad aplicada a la educación del siglo $X X I$}

Los sistemas educativos en España siempre han enfocado la enseñanza necesaria para la formación del alumnado en la división del tiempo lectivo en una serie de materias diferenciadas e inconexas; sin embargo, nada hay más alejado de la realidad. Esto ha provocado la limitación y segregación de las mismas, y esa monodisciplinariedad es, según Juntsch (1979), un principio estático que pierde sentido y efectividad cuando se pretenden abordar objetivos, especialmente en el ámbito educativo, cuya finalidad es desarrollar un pensamiento interdisciplinar. $Y$ es que es imposible comprender la economía sin la colaboración de la matemática 
o la matemática sin la comprensión de las formas que representa en muchas de sus áreas de estudio. Estos dos ejemplos sencillos son sólo una pequeña muestra de la imposibilidad de dividir de modo férreo el conocimiento en materias aisladas.

En otra línea que apoya el mismo enfoque, Chacón Reyes (2006) nos centra la atención a cerca del hecho indiscutible de que la sociedad en cualquier momento de la historia es consecuencia de la situación y los cambios producidos en una totalidad interrelacionada y no en hechos puntuales inconexos. Si una de las funciones principales de la educación es transmitir la herencia cultural y sus valores será imposible hacerlo desde perspectivas aisladas puesto que, señalando un ejemplo sencillo, la evolución en el campo de la óptica durante el siglo XVIII que propició la evolución en el microscopio inventado por Galileo desembocó en una revolución sin precedentes en el campo de la biología y medicina, de consecuencias que no es necesario relatar aquí.

La interconexión de las disciplinas es evidente, pero de modo paralelo nos enfrentamos a la problemática de la súper-especialización debida al inmenso avance de la ciencia, que como expresan Pozuelos Estrada, Rodríguez Miranda y Travé González (2012) "amenaza con dislocar y fragmentar las estructuras culturales en parcelas inconexas de escasa comprensión y utilidad" (p.563). Esto sólo puede reconducirse desde el punto de vista de reconectar las disciplinas, no se trata de que desaparezcan si no de que cobren un sentido más amplio dentro de una cierta estructura común. Como apunta Egg (1994), hasta el Renacimiento el universo era considerado un todo indisoluble, concepto que se pierde con el desarrollo de la ciencia.

Según las definiciones aportadas por este autor, se tratará aquí de sustituir la pluridisciplinariedad no tanto por una transdiciplinariedad como por una interdisciplinariedad. No se trata de eliminar totalmente los límites de las disciplinas con la idea de unificarlas sino de dominar la propia disciplina, pero teniendo conocimientos de las otras implicadas. Para Morin (2014), el concepto de interdisciplina se refiere a la transferencia de métodos entre las disciplinas y la multidisciplina consiste en juntar disciplinas para conocer objetos desde las diferentes perspectivas, comenzando a desdibujar las fronteras entre los distintos ámbitos. Sin embargo, la transdisciplina alude al diálogo y la complejidad de mezclar las disciplinas, eliminando las barreras que la sociedad ha establecido previamente. Desde la perspectiva de De Souza (2009), transdisciplinaridad es ver el todo desde una perspectiva única, aunque múltiple, que podría suponer tanto en educación e innovación un sistema coordinado de niveles y objetivos múltiples (Juntsch, 1979). Esta idea se aproxima al concepto de competencias que se refiere a aquellos aprendizajes básicos para el desarrollo integral de un alumno desde todas las áreas de conocimiento, tal y como se define en el Real Decreto 1631/2006, de 29 de diciembre, de educación (BOE núm. 5, viernes 5 enero 2007). Estas competencias, aunque regulan la etapa de la ESO, se deben seguir trabajando durante la etapa de Bachillerato para reforzar el desarrollo del alumno 
y por qué no en la educación universitaria, donde hay un mayor predominio de la monodisciplinariedad.

Si hablamos de educación, seguramente nos dirigimos hacia la nueva idea del currículum integrado en el sentido que define Torres Santomé (2006), como estudios interdisciplinares en un contexto globalizado que doten de significado a los contenidos. Paralelamente se debe resolver la adaptación a las peculiaridades motivacionales del alumno, y esta cuestión parece más abordable desde una enseñanza menos parcelada que muestre las correlaciones entre las disciplinas. Pero, además, dotar de la capacidad para responder a los problemas desde puntos de vista correspondientes a distintas áreas del conocimiento aportará una versatilidad y competencia fundamentales en los nuevos entornos laborales, como se expondrá en el apartado siguiente. Delgado (2009) también presenta cómo el siglo XXI va a estar caracterizado por una globalización compleja repleta de interconexiones por lo que la sectorización del conocimiento no va a ser posible. Redunda en la idea de la evolución social como un todo coherente que alcanza una dimensión mucho más constatable con la evolución de las nuevas tecnologías que facilitan los procesos interactivos y las posibilidades de transferencia de información.

A la vista de todo lo expuesto y de acuerdo con Martínez, Perera, Álvarez, Lugo y Boza (2011), debemos aproximarnos a una enseñanza interdisciplinar partiendo de la organización en currículos fragmentados, puesto que este es el contexto educativo con el que nos encontramos y es en él donde se están dando experiencias docentes en este sentido. Estos autores plantean la posibilidad de establecer un sistema gráfico matricial contenidos y asignaturas para establecer su interrelación, paralelamente, este sistema podría aplicarse a la metodología propuesta relacionando los contenidos de Expresión Gráfica, Visual y Audiovisual con los ámbitos profesionales propuestos.

Sin olvidar la realidad de la fragmentación en materias establecida por la legislación, no podemos obviar que los cambios en los últimos años suponen un acercamiento al camino de la interdisciplinariedad. La introducción en nuestro sistema educativo a partir de la Ley Orgánica 2/2006 de 3 de mayo de Educación, del concepto de educación en competencias y su definición, tema que será tratado en profundidad en apartados posteriores, junto a la inclusión de contenidos transversales educativos nos aproximan a un modo distinto de entender la formación escolar. Esto ha conllevado nuevas exigencias a la labor docente y una creciente preocupación acerca de su formación: como describe Vezub (2007), el docente se enfrenta a los nuevos retos educativos consecuencia de la sociedad globalizada del siglo XXI.

En gran medida, uno de los problemas que plantean las propuestas a cerca de la interdisciplinariedad es que debería abordarse en un primer estadio modificando la formación del profesorado y basando la enseñanza en un sistema 
cooperativo. Delgado (2009) no obvia la problemática que supone plantear procesos interdisciplinarios en el aula, tanto a nivel individual como cooperativo: exigiría el dominio básico de varias áreas del conocimiento y saber ver la materia desde las partes hasta la globalidad. Por estos motivos, supone un camino difícil y que exige la formación de equipos de trabajo con mucha capacidad colaborativa.

\section{La enseñanza multidisciplinaria, interdisciplinaria y transdisciplinaria}

Los aspectos que se deben desarrollar mediante la inclusión de las competencias, según Sierra, Méndez-Giménez y Mañana-Rodríguez (2013), no sólo deben estar vinculados a los objetivos, contenidos y actividades, sino que también implican una metodología más interdisciplinar y globalizada, con una clara tendencia a un propósito común en el sistema (Juntsch, 1979). De esta forma la inclusión del concepto interdisciplinariedad en la enseñanza viene motivada por la necesidad de entender que para desarrollar una competencia es necesario ejercitarla desde diversos contextos y también en la etapa de Bachillerato.

Precisamente las tendencias actuales de innovación desarrollan y crean conceptos científicos interdisciplinares, donde se exige un proceso reflexivo y crítico sobre cómo se representa, describe, comprende, explica y modifica la realidad, conceptos inabordables desde una única disciplina (Fernández-Ríos, 2010). El término exacto que, desde estas aproximaciones se pretende desarrollar en el trabajo, es la multidisciplinariedad para poder aproximarse a los objetos geométricos desde distintos ámbitos simultáneamente. De este modo, se puede demostrar que los distintos lenguajes tienen bases comunes y diferentes formas de acercarse a un concepto pero que son complementarias.

De acuerdo con las propuestas de Sierra et al. (2013) es imprescindible establecer conexiones y actividades interdisciplinares, que eviten la compartimentación estanca de los contenidos, entre las distintas disciplinas. Disciplinas que, según Fernández-Ríos (2010), tienen tres razones de ser: cultural, epistemológica y de lenguaje, influyendo esta fragmentación del conocimiento en nuestra forma de entender la realidad de forma inconexa. En este contexto, el docente se presenta como la persona que introduce contenidos en el aula, desde diferentes enfoques que se comuniquen, procurando que los discentes adquieran una visión más amplia y profunda de los conceptos.

La demanda actual exige profesionales que tengan capacidad de profundizar en saber, aprender a aprender y en poder trabajar de forma cooperativa, es decir, que tengan capacidad de resolver problemas eliminando los límites de las disciplinas. En otras palabras, las denominadas competencias que se incluyeron en el sistema educativo tienen su razón de ser en las demandas del mercado laboral, por eso también son importantes en la etapa de Bachillerato o ciclos formativos (Montoro, Morales y Valenzuela, 2014) y en la universitaria. Como las competencias abordan la complejidad de la realidad, ahora hay una necesidad 
de abordar los problemas desde varias miradas monodisciplinares (Meinardi, Adúriz y Revel, 2002) y ello enraíza en los valores socialmente compartidos desde diferentes ángulos.

También, se debe tener en cuenta que existe un cierto grado de libertad en la interdiciplinaridad, ya que se debe permitir al alumno que explore con más profundidad aquellos ámbitos de conocimiento que llamen su atención y le interesen (Oliva, 2003). Es importante destacar que este tipo de aprendizaje se propicia en el intercambio de información, por lo que, viviendo en la sociedad actual, la interdisciplinaridad debería ser un hecho. Meinardi et al. (2002) señalan que, tanto en la teoría como en la práctica didáctica de las ciencias experimentales, existe desde el inicio una gran tendencia a enfocar el aprendizaje de forma interdisciplinar.

Otras materias de la educación, como la Educación Ambiental o las Tecnologías, ya se construyen combinando diversas disciplinas dentro de una nueva concepción multidisciplinar para la educación científica (Meinardi et al., 2002). Esta situación es extrapolable a la asignatura de Dibujo Técnico, aunque todavía no se ha puesto en práctica. Este hecho es un reflejo de cómo es la realidad actual, el conocimiento se interrelaciona entre las distintas disciplinas. Precisamente la necesidad de este tipo de enseñanza se nutre de las nuevas tecnologías de la información, ya que son herramientas que permiten el acceso al conocimiento y eliminan los límites que las propias disciplinas se imponen (Oliva, 2003).

Para eliminar estas barreras entre disciplinas será necesario re-elaborar y re-pensar los modelos y métodos de enseñanza e incorporar las TIC de forma aplicada. Deesta forma se conseguirá profundizar en la adquisición de conocimiento múltiple, especialmente en la materia de objeto de este trabajo. Para el caso del Dibujo Técnico, establecer relación con las Matemáticas es un sencillo paso de multidisciplinariedad entre conceptos complementarios. Un claro ejemplo de aplicación es la utilización de la imagen como ayuda para mejorar la comprensión y la resolución de problemas matemáticos como demuestran Vicente, Orrantia, y Verschaffel (2008).

\section{La asignatura de enseñanza y aprendizaje de la expresión plástica y artística en el grado de educación infantil}

Esta asignatura de tercer curso del Grado de Educación Infantil de una universidad española tiene entre sus objetivos "Conocer y experimentar las posibilidades del lenguaje plástico como medio de expresión y de conocimiento para desarrollar la capacidad creadora". De acuerdo con ello, se pretende formar al alumnado en la programación de actividades, procesos de trabajo y técnicas adecuadas para la Educación Infantil desde el lenguaje artístico plástico. Con el fin de cumplir con estos objetivos y desarrollar competencias como la creatividad y el trabajo en equipo, de manera interdisciplinar se propuso al alumnado la realización de una 
serie de prácticas en las que combinasen la expresión plástica trabajada en esta asignatura con la expresión corporal, con la música, el lenguaje y las inteligencias múltiples. En este último sentido, se partió de la consideración de Rodríguez (2014), que consideraba las actividades lúdicas y de experimentación como la mejor forma de potenciar su desarrollo; de ahí, la importancia de la estructuración de las actividades propuestas con el "toque" multidisciplinar.

Las actividades desarrolladas se comprendían dentro de los contenidos teóricos de la citada materia, "Enseñanza y Aprendizaje de la Expresión Plástica y Artística" del tercer curso del Grado de Educación Infantil. La finalidad de estas actividades interdisciplinares que se realizaron era dar a conocer a los discentes el valor de una educación integral en la etapa de Educación Infantil y la necesidad de trabajar de manera conjunta, a partir de los distintos lenguajes del área de comunicación y representación. Música y Plástica deben comprenderse como lenguajes que interaccionan, de acuerdo con el camino abierto por especialistas como Gutiérrez-Cordero y Díaz-Alcaide (1997), que a finales del siglo XX insistieron en la importancia de esta interacción en la formación del futuro profesorado.

La Educación Artística ayuda al sujeto a canalizar sus emociones a través de unos medios de expresión con carácter artístico. Esto posibilitó unos ejercicios interdisciplinares cuya meta era salir, ocasionalmente, del aula para huir de la clásica metodología magistral e incentivar que el alumnado utilizase las artes plásticas y visuales como fuente y núcleo integrador de experiencias transversales, creando situaciones de aprendizaje a través de éstas. Así pues, la finalidad de esta materia no fue únicamente enseñar al futuro maestro a pintar, escenificar o dibujar aisladamente, sino que se posibilitó el descubrimiento del mundo a través de un "motivo" o una razón que se convirtió en importante para él gracias a la interacción disciplinar (y personal). De esa forma, el futuro docente podrá transmitir al educando todo su saber y conocimiento a través de experiencias manipulativas y sensoriales, siempre contando con innumerables materiales que favorezcan aprendizajes significativos (Moreno, 2015).

Asimismo, aunque no forma parte de los contenidos de este artículo, no podemos dejar de comentar que dentro de esta asignatura se trataron otros temas centrados en la representación bidimensional y tridimensional de los niños, las etapas gráficas del dibujo infantil, el trabajo de distintas propuestas didácticas partiendo del currículum vigente, y distintas propuestas de cómo puede trabajarse el patrimonio y la Historia del Arte en niños de 0 a 6 años. Así, a lo largo del desarrollo de las unidades se combinaron teoría y práctica, tanto dentro como fuera del propio campus universitario, combinándose siempre con un adecuado uso de las TIC; también, se recurrió a programas para maquetar cuentos, se realizó una visita a un museo, se trabajó con diferentes materiales y técnicas artísticas, etc. El profesorado recogió todo este trabajo en un blog digital de la asignatura, en el que también participó todo el alumnado subiendo sus trabajos; aunque, no fue el objetivo principal de la experiencia que se narra en este artículo. 


\section{Metodología}

\section{Propuesta metodológica}

Basándonos en la idea de unir educación integral e interdisciplinariedad se planteó a todo el alumnado de la asignatura artística, que cursaban también las materias de "Psicología del Desarrollo", "Enseñanza y Aprendizaje de la Expresión Musical" y "Enseñanza y Aprendizaje de la Lengua Castellana", las siguientes tres actividades: 1) elaboración y dramatización de cuentos infantil, 2) taller sobre arte, música y cuerpo y 3) Desarrollo de las inteligencias múltiples a través de recursos plásticos. Las tres se centran en una serie de competencias específicas y competencias transversales a trabajar en cada una de ellas, tal y como se exige en las materias de los grados universitarios, concretamente en el Libro Blanco para el título de Grado en Magisterio y la Orden ECl 3854 de 2007 para el Grado de Educación Infantil. Entre ellas, las competencias transversales que se trabajaron fueron:

- T10 Trabajo en equipo de carácter interdisciplinar.

- T18 Creatividad.

- T1 Capacidad de análisis y síntesis.

- T6 Capacidad de gestión de la información.

Mientras que, respecto a las competencias específicas, todas ellas están relacionadas con las asignaturas implicadas y que son:

- E56 Elaborar propuestas didácticas que fomenten la percepción y la expresión musical, las habilidades motrices, el dibujo y la creatividad.

- E58 Promover la sensibilidad relativa a la expresión plástica y a la creación artística.

- E1 Comprender los procesos de aprendizaje relativos al periodo 0-6, en el contexto familiar, social y escolar.

- E6 Conocer la dimensión pedagógica de la interacción con los iguales y los adultos y saber promover la participación en actividades colectivas, el trabajo cooperativo y el esfuerzo individual.

- E50 Conocer y utilizar adecuadamente recursos para la animación a la lectura y a la escritura. 


\section{Actividad 1. Elaboración y dramatización de cuentos infantiles}

Esta primera actividad consistió en la elaboración de un cuento para Infantil en lengua castellana y en la maquetación en forma de libro con imágenes e ilustraciones con el programa de diseño gráfico llamado Indesign (Adobe Collection). Ese recurso digital fue propuesto porque su manejo abre la posibilidad a los alumnos de poder elaborar otro tipo de fichas didácticas y actividades para el aula e utilizar al mismo tiempo las nuevas tecnologías. El resultado fueron auténticos libros de imprenta debido al alto nivel en el diseño y ejecución de sus imágenes y caligrafía.

La segunda parte de la actividad era crear la escenificación (escenario, puesta en escena y personajes) de cada uno de los cuentos siempre utilizando técnicas y recurso artísticos estudiados a lo largo de la materia y así poner en práctica algunos de los contenidos vistos. La temática era libre, pero siempre adecuada a niños entre 3 y 6 años y vinculada a los bloques conceptuales del 20 ciclo de Educación Infantil, y realizada por los discentes en grupos de seis personas. Para su desarrollo, escogieron la realización de títeres, marionetas, sombras chinescas e incluso la representación teatral a través de disfraces. Esto ocasionó una gran variedad de propuestas para las dramatizaciones lo que hizo mucho más interesante y amena la propuesta.

\section{Actividad 2. Taller sobre arte, música y cuerpo}

Dentro de la práctica con la asignatura de "Enseñanza y Aprendizaje de la Expresión Musical" se propuso un ejercicio práctico en el gimnasio y de esa forma 1) ir más allá de las manualidades, incorporando nuevas expresiones artísticas en la que todos participen, incluido el profesor y 2) emplear una gran variedad de técnicas y procesos que desarrollen distintas capacidades del alumno. También, se fomentó la interdisciplinar al introducirles antes de cursarla en contenidos vinculados a la música y el ritmo.

La propuesta metodológica contó con la colaboración de otro docente, especialista, en Música y Musicoterapia. Una vez ubicados en un espacio diáfano y cómodo se procedió a la realización de una serie de ejercicios de carácter grupal (2-3 miembros, grupos de 6, grupos de 60 alumnos) y otros individuales que buscaban la relajación y la reflexión de cada discente.

Durante el taller se emplearon una gran variedad de recursos, entre los que destacaron: las audiciones, los juegos de respiración y de relajación, la distinción y la diferenciación de sonidos y ruidos, la educación rítmica y las actividades basadas en el lenguaje corporal. Estas últimas trataron de explorar y disfrutar con el cuerpo con acciones inverosímiles impulsadas por un espíritu creativo y por el juego en sí mismo. 
Entre las actividades trabajadas que permitieron comprender el importante trabajo interdisciplinar realizado, en pos de favorecer la educación integral del educando, así como la formación integral del futuro docente, fue el trabajo corporal de puntos, líneas y espacios. Estos conceptos tan plásticos fueron plasmados corporalmente con un acompañamiento musical que iba variando, de cara a potenciar la atención y la sincronización cuerpo-música (como concepto más puramente musical) (Gilabert y Bernabé, 2016).

Otro ejercicio interesante y que permitió un trabajo claramente interdisciplinar fue la expresión de sentimientos y emociones con determinadas partes del cuerpo, en respuesta a piezas musicales seleccionadas. En lugar de perseguir una plasmación gráfica sobre el papel de una emoción, se persiguió la plasmación corporal de una emoción que el cuerpo del compañero debía reflejar igualmente (Gilabert y Bernabé, 2016).

\section{Actividad 3. Desarrollo de las inteligencias múltiples a través de recursos plásticos}

Este ejercicio consistía en trabajar uno de los teoremas estudiados en la asignatura de Psicología del Desarrollo. Se trata de la teoría de las inteligencias múltiples por el que Howard Gardner recibió el Premio Príncipe de Asturias de Ciencias Sociales en el año 2011. Según este especialista, cada persona tiene, por lo menos, ocho inteligencias o habilidades cognoscitivas: musical, cinético-corporal, lógicomatemática, lingüística, espacial y visual, interpersonal, intrapersonal y naturalista (esta última fue añadida posteriormente). En concreto, "la inteligencia visual y espacial explica que los niños y niñas piensan en imágenes y dibujos, tienen facilidad para resolver rompecabezas, dedican el tiempo libre a dibujar, prefieren juegos constructivos, etc.; pero todas ellas se pueden trabajar de forma conjunta a través de recursos plásticos" (Gilabert González, 2016, p. 44).

Para su elaboración de los grupos de 6 personas debía escoger dos como mínimo de las ocho inteligencias múltiples para trabajar a través de un recurso plástico o visual que fue confeccionado en las clases de Plástica. Así se presentó a los alumnos una relación, a modo de ejemplo, para sugerirles posibles propuestas reales:

- Inteligencia lingüística: teatro, programas de radio o grabaciones.

- Inteligencia lógico-matemática: aprendizaje cooperativo y uso de juegos lógicos.

- Inteligencia espacial: trabajos artísticos, mapas geográficos, mapas mentales y puzles.

- Inteligencia corporal-kinestésica: tocar y sentir los propios aprendizajes.

- Inteligencia musical: cantar, escuchar música, tocar instrumentos. 
- Inteligencia naturalista: contacto con la naturaleza y los fenómenos naturales.

- Inteligencia intrapersonal: proyectos individuales y diarios personales.

- Inteligencia interpersonal: aprendizaje cooperativo, debates y tutorías entre iguales.

En la asignatura de "Psicología del Desarrollo" recabaron información sobre las inteligencias múltiples escogidas siguiendo las pautas marcadas por el profesor en cuestión. Por el contrario, en la parte plástica realizaron el diseño de la actividad donde tenían que explicar el recurso elegido, justificar la elección y cómo iba a ser realizado (brevemente hablar de los pasos a seguir y los materiales escogidos para su elaboración). Por último, grabaron un vídeo en el que los propios alumnos ponían en práctica con niños el o los recursos plásticos propuestos y de esta forma poner en uso las nuevas tecnologías, ya que tenía que subir el vídeo en el canal youtube, y al mismo tiempo ver el correcto manejo del recurso y su verdadera utilidad y eficacia en los discentes de Infantil.

\section{Resultados y discusión}

En el caso de Música, la legislación vigente para Educación Infantil, ya citada, establece que debe trabajarse el lenguaje artístico, mostrando unidos el Plástico y el Musical. De modo que, desde las dos asignaturas del Grado de Educación Infantil, se pueden trabajar de forma conjunta para mostrar su carácter inseparable, aunque característico e independiente al mismo tiempo. Además, el Real Decreto 1630/2006 establece una serie de características para los lenguajes plástico y musical y que en la actividad propuesta fueron tratados de manera conjunta. Por ejemplo, la manipulación de objetos e instrumentos con espontaneidad expresiva para adquirir nuevas destrezas y sensibilidades fue puesta en práctica con una selección de músicas de fondo que sirvieron como estímulo para el proceso creativo. Asimismo, el despertar de la sensibilidad estética que surge de la escucha atenta, se potenció al estimularlos a dramatizar escenas con movimiento detenido, como si se tratase de un cuadro tridimensional.

Por un lado, el alumnado se mostró muy activo durante la realización de las distintas actividades, comprendiendo la importancia de unir materias principales que en el caso de la plástica y la música constituyen en la educación obligatoria el área de Educación Artística. Aunque en Infantil no se encuentre esa organización, resulta significativo orientar al futuro profesorado a trabajar en esa línea interdisciplinar, ya que sólo así el aprendizaje del alumnado será comprendido por éstos como un proceso global donde todo conocimiento depende de otro y donde, y esto es lo más importante para el docente, se conseguirá una formación completa del educando como ser humano social y cultural (y artísticamente) competente. 
Además, en los casos de Lengua Castellana y Psicología del Desarrollo fue muy importante plantear estas conexiones a través de prácticas colaborativas porque ayuda a los discentes a crear conexiones entre las distintas asignaturas que a simple vista parecen no tener relación alguna. Como se puede comprobar, desde las cuatro materias se puede trabajar de manera unísona una serie de competencias transversales y específicas que deben ser alcanzadas por los discentes en el Grado de Educación Infantil.

Por otro lado, esta experiencia no sólo fue beneficiosa para los docentes en formación, sino para los docentes encargados de colaborar en una misma clase. El profesorado universitario parece obviar la necesidad de mostrar y demostrar a su alumnado esa necesidad de trabajar con el compañero, ese enriquecimiento que supone la práctica compartida. El profesorado en ciernes nunca podrá trabajar en equipos docentes sin haber experimentado previamente el enriquecimiento que esto supone para su práctica docente.

Sobre otro aspecto, estos ejercicios interdisciplinares tienen como finalidad que el alumno establezca las conexiones intrínsecas entre los diferentes contenidos porque, como sugiere Fernández-Ríos (2010), la complejidad de la naturaleza y la sociedad no está sujeta a la monodisciplinariedad. Por ello, según Jurado (2009), es necesario que exista una perspectiva multidimensional, que reconozca la pluralidad implícita en la heterogeneidad que existe hoy. Este será un requerimiento imprescindible en los años sucesivos para hacer frente a las nuevas exigencias, tanto en la vida social como en la académica. Continuando con esta idea, Oliva (2003) apunta a la necesidad de un sistema interdisciplinar donde surjan intercambios de conocimientos entre diversas disciplinas, requisito ya expresado por la Unesco en 1998. Y será el enfoque multidisciplinar el que asegure el logro de la excelencia en educación y en la docencia y la investigación.

Precisamente la necesidad de este tipo prácticas interdisciplinares se pueden nutrir de las nuevas tecnologías de la información, ya que son herramientas que permiten el acceso al conocimiento y eliminan los límites que las propias disciplinas se imponen. Las tecnologías serán, como indican Mendoza y Herrera (2012), mediadoras y transformadoras del modelo educativo actual y propiciarán nuevas prácticas que permitan el tratamiento automático de la información. Además, es muy importante poseer conocimientos tecnológicos para poder incorporarse a la vida laboral y comprender los medios digitales que se actualizan de forma continua. De acuerdo con esta tendencia, será importante otorgar más protagonismo a los conocimientos tecnológicos desde la educación universitaria y es un recurso tan versátil que puede emplearse en cualquier disciplina y para cualquier contenido. 


\section{Conclusiones}

En definitiva, respecto a la Plástica, se demostró que las prácticas artísticas brindan un beneficio social y estimulan el desarrollo integral del ser humano como individuo y su relación con el entorno. Es más, su actividad le provoca, al menos en el tiempo de elaboración y ejecución, una sensación placentera y de felicidad. Asimismo, a través del arte, el hombre desde niño expresa emociones, ideas, valores y su visión crítica del mundo (Gilabert González, 2016). Sin embargo, a pesar de su gran valor, la Plástica se ha descuidado e infravalorado en el sistema educativo español de tal modo que la carencia de una educación artística correcta y con sentido integral es casi absoluta (Sánchez Alarcón, 2000). Es más, técnicas como las teatrales brindan recursos para enriquecer la espontaneidad, la escucha activa, la afectividad, la exploración, y el juego con la voz y el cuerpo, pero ni tan siquiera se plantean como recurso educativo en los distintos niveles o etapas.

Esta propuesta metodológica demuestra que la expresión plástica y la expresión musical son un lenguaje más, un medio más de representación y un instrumento básico de comunicación. Comunicarse a través del dibujo, del volumen, de la forma, del ritmo, de la melodía... Y que, a su vez, pueden convertirse en un recurso educativo, que permita comprender los contenidos del resto de áreas de una manera global, de ahí su importancia en el proceso de enseñanza y aprendizaje de los futuros docentes; puesto que, su objetivo en la escuela es favorecer el desarrollo integral, la percepción, la creatividad, la autoestima, además del desarrollo cognitivo que siempre se prioriza en detrimento de los anteriores. Asimismo, es muy importante fomentar este tipo de iniciativas entre las materias de las carreras universitarias tanto del sector público como privado, ya que se necesitan hoy y en el futuro profesionales versátiles e interdisciplinares. 
La formación del futuro docente a través de la interdisciplinariedad y la educación integral

por Luz Ma Gilabert González y Ma del Mar Bernabé Villodre

\section{Referencias bibliográficas}

Barrientos, P. (2013). Visión integral de la educación. Horizonte de la Ciencia,3 (4), 61-65.

Chacón Reyes, J. (2006-2007). Educación, interdisciplinariedad y pedagogía. Pampedia, 3, 22-26.

Chao, R., Mato, Mạ D., \& López, A. (2015). ¿Se trabajan de forma interdisciplinar música y matemáticas en educación infantil? Educaçao e Pesquisa, 41(4), 1009-1022.

De Souza, B.B. (2009). Hibridación y transdisciplinariedad en las artes plásticas. Educación Siglo XXI, 27(1), 217-230.

Delgado, R. (2009). La integración de los saberes bajo el enfoque dialéctico globalizador: la interdisciplinariedad y transdisciplinariedad en educación. Investigación y Postgrado, 24(3), 11-44.

Egg, A. (1994). La interdisciplinariedad en educación. Recuperado de https://fido. palermo.edu/servicios_dyc/blog/docentes/trabajos/41974_158754.pdf

Fernández-Ríos, L. (2010). Interdisciplinariedad en la construcción del conocimiento: ¿Más allá de Bolonia? Innovación educativa, 20, 157-166.

Gervilla, E. (2000). Un modelo axiológico de educación integral. Revista española de pedagogía, 215, 39-58.

Gilabert González, L.M. (2016). La educación artística y la expresión plástica infantil: qué y cómo enseñar. Madrid: Dikynson.

Gilabert González, L.M. y Bernabé, M. (2016). Interdisciplinariedad y Educación Integral: una propuesta de formación desde la Educación Artística en Infantil. En T. Ramiro-Sánchez, \& Ma T. Ramiro (Coord.), Avances en Ciencias de la Educación y del Desarrollo (pp. 717-721). Granada: Asociación Española de Psicología Conductual.

Gutiérrez-Cordero, R., \& Díaz-Alcaide, Mạ D. (1997). Música y plástica: su interacción en la formación del profesor. Revista de Enseñanza Universitaria, 1, 119-225.

Jurado, F. (2009). El enfoque sobre competencias: Una perspectiva crítica para la educación. Revista Complutense de Educación, 20(2), 343-354.

López-Peláez, Mạ P. (2010). El papel de la música y las artes en una educación integral. Arte y movimiento, 3, 37-44. 
Juntsch, E. (1979). Hacia la interdisciplinariedad y la transdisciplinariedad en la enseñanza y la innovación. En L. Apostel, G. Berger, A. Briggs, \& G. Michaud (Ed.), Interdisciplinariedad. Problemas de la enseñanza y de la investigación en las universidades (pp. 110-141). México: ANUIES.

Martínez Rubio, B.N., Perera Curmema, F., Álvarez Pérez, M., \& Boza Oramas, Y. (2011). La interdisciplinariedad en el proceso aprendizaje-enseñanza de las ciencias, La Habana: Educación Cubana.

Meinardi, E., Adúriz, A.,\& Revel, A. (2002). La Educación Ambiental en el aula. Una propuesta para integrar contenidos multidisciplinares a través de la argumentación. Investigación en la Escuela, 46, 93-103.

Mendoza, N.E., \& Herrera, L. (2012). Herramientas y tendencias para la enseñanza apoyada por TIC: docencia y líneas de investigación. Virtualis, 3(6), 42-57.

Montoro, J., Morales, G.P., \& Valenzuela, J.R. (2014). Competencias para el uso de tecnologías de la información y la comunicación en docentes de una escuela normal privada. Virtualis, 5(9), 21-33.

Moreno, F.M. (2015). La utilización de los materiales como estrategia de aprendizaje sensorial en infantil, Opción, 31(2), 772-789.

Morin, E. (2014). ¿Qué es Transdisciplinariedad? Recuperado de http://www.edgarmorin.org/que-es-transdisciplinariedad.html

Oliva, M.A. (2003). El espacio europeo de educación superior: una oportunidad de desarrollo multidisciplinar a través del aprendizaje y la tecnología. Encuentros multidisciplinares, 5(15), 46-54.

Pérez, S., \& Leganés, E.N. (2012). La Música como herramienta interdisciplinar: un análisis cuantitativo en el aula de Lengua Extranjera de Primaria. Revista de Investigación en Educación, 10(1), 127-143.

Piaget, J. (1990). El nacimiento de la inteligencia. Barcelona: Crítica

Pozuelos Estrada, F., Rodríguez Miranda, F., \& Travé González, G. (2012). El enfoque interdisciplinar en la Enseñanza universitaria y el aprendizaje basado en la investigación. Un estudio de caso en el marco de la formación. Revista de Educación, 357, 561-585.

Rodríguez, F. (2014). Una o múltiples inteligencias. Padres y Maestros, 357, 6-9.

Rodríguez, W. (2012). La educación integral o el ideal educativo burgués. Miscelánea Comillas, 70(137), 517-532. 
Sierra, B., Méndez-Giménez, A., \& Mañana-Rodriguez, J. (2013). La programación por competencias básicas: hacia un cambio metodológico interdisciplinar. Revista complutense de educación, 24(1), 165-184.

Torres Santomé, J. (2006). Globalización e interdisciplinariedad: el currículum integrado. Madrid: Morata.

Vezub, L.F. (2007). La formación y el desarrollo profesional docente frente a los nuevos desafíos de la escolaridad. Profesorado: Revista de currículum y formación del profesorado, 11(1), 1-23. 